\title{
Comparação entre Ferramentas CASE para gerenciamento de Projeto e Métricas de Software no Curso de Sistemas da Informação do UniFOA
}

\author{
Professor Doutor Jason Paulo Tavares Faria Junior (Sistemas da Informação - UniFOA) - \\ jason-rj@bol.com.br
}

\section{Resumo}

O presente artigo apresenta uma análise comparativa entre diferentes ferramentas que automatizam a aplicação de métricas de software, sendo resultado de um projeto que visa a implementação de uma ferramenta CASE para Gerenciamento de Projeto e métricas de Software no Curso de Sistemas da Informação do UniFOA.

Palavras chave: Gerenciamento de Projeto ; Métricas de Software; Ferramenta CASE

\section{Introdução}

Um dos objetivos do projeto é estudar algumas ferramentas CASE para métricas de software e efetuar a análise comparativa visando detectar as diferentes funcionalidades implementadas como forma de embasar e ampliar os conhecimentos para o desenvolvimento da ferramenta CASE que resultará do projeto. Detectou-se que são poucos os softwares existentes, atualmente no mercado, que viabilizam a medição de software. E cada software apresenta um conjunto de características diferentes, que estão relacionadas neste artigo.

\section{Métricas de Software}

Quando se considera boa parte dos empreendimentos técnicos, verifica-se que as medições e as métricas permitem um melhor entendimento do processo utilizado para desenvolver um produto, assim como uma melhor avaliação do próprio produto, consenso encontrado em Candéas (1999) e Pressman (1995). A quantificação dos aspectos relacionados ao processo de obtenção de um produto, assim como do produto, é importante, pelas seguintes razões:

- No caso do processo de desenvolvimento, as medições podem permitir melhorias no processo, aumentando a sua produtividade;

- No caso do produto, as medições podem proporcionar informações a respeito de sua qualidade.

\section{Ferramentas CASE de métricas de software}

Esta seção apresenta uma descrição sucinta de diferentes ferramentas que implementam métricas de software. As ferramentas analisadas são:

- Costar 
- USC-COCOMO

- Calico

- Cost Xpert 2.1

\subsection{Costar}

Costar é um Software de Gerenciamento de tempo, custos e avaliação de métricas para desenvolvimento de projetos, visualizado na figura 1. Este software foi desenvolvido pela Softstar Systems, cujo site é http://www.softstarsystems.com. A versão pesquisada foi desenvolvida em 1997 e, atualmente, encontra-se na versão 5.0, sendo que, para a análise foi utilizada uma versão demo cuja única limitação é o valor máximo permitido para o $\mathrm{PF}$, tendo sido estabelecido um limite máximo de 500 pontos.

A principal função do Costar é estimar o esforço (pessoas) e o tempo e custo de projetos, através da interligação de tarefas e pessoas. No que se refere ao esforço são passados os dados de experiência, o cargo exercido, e o custo de determinada pessoa.

No que se refere a processo a ferramenta padroniza alguns modelos predefinidos. Com base nos modelos apresentados o usuário pode alterar o início da iteração (etapa) seguinte, no entanto, não é permitido ao usuário configurar etapas diferenciadas.

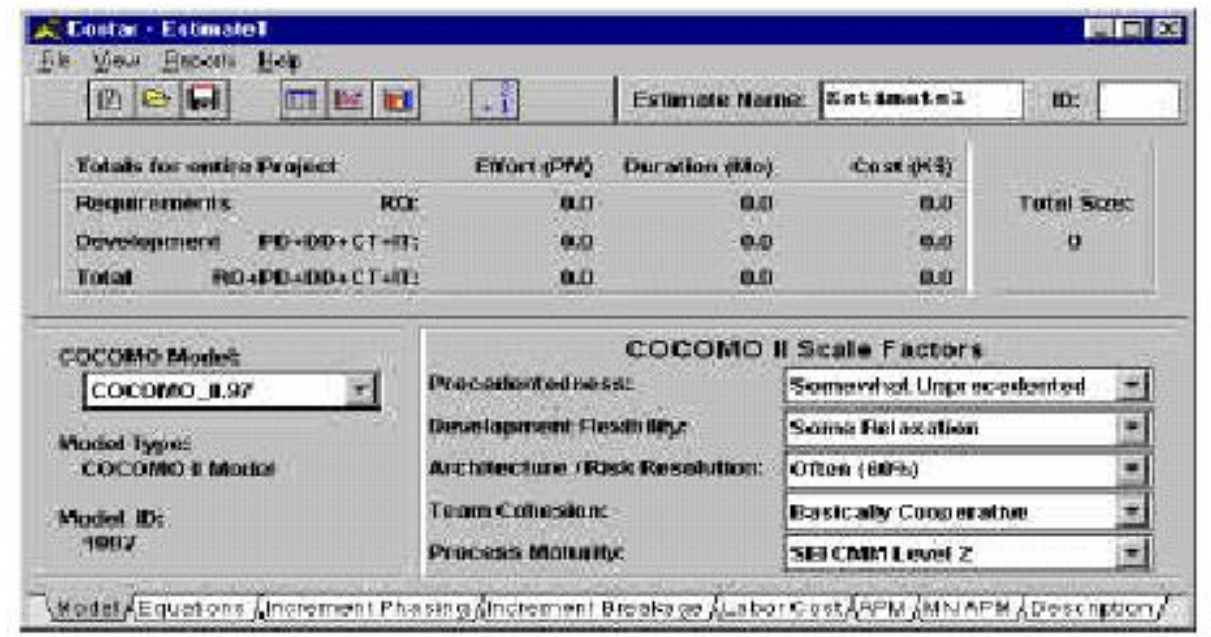

Figura 1-Ferramenta Costar

No que tange ao esforço (pessoal) as informações requeridas (consideradas) são as experiências em máquina, linguagem, programas, plataformas, bem como, o horário de desenvolvimento, relação de tempo, dentre outras.

Quanto às informações pertinentes ao produto o software considera para o cálculo: tamanho de banco de dados, complexidade do produto, requerimento de recurso, dentre outros.

Para a geração das estimativas são consideradas todas as informações anteriormente citadas (exigências do desenvolvimento), tendo-se como resultado informações referentes a: esforços (em pessoas/mês), duração (meses) e custos (K\$).

O software também fornece recursos para visualização gráfica, apresentando a distribuição de pessoal, marcos de referências e também alguns relatórios detalhados. Tais relatórios contextualizam, de maneira textual, informações referentes a esforço, o custo e a duração (tempo). 


\subsection{Calico}

O CALICO é um software que executa calibrações de equações do modelo COCOMO. Esta ferramenta foi desenvolvida pela empresa Softstar Systems, a mesma descrita na seção 4.1, sendo que, o CALICO vem sendo utilizado para calibrar equações tanto no modelo COCOMO tradicional, como no modelo COCOMO II. A versão pesquisada foi desenvolvida em 1999 e, atualmente, encontra-se na versão 5.06, de modo que, para a análise foi utilizada uma versão demo cuja única limitação é o fato de não possuir um Help on-line, para guiar seus usuários. Baseado em dados referentes ao projeto (linguagem de programação utilizada, custo por mês dos integrantes do projeto, horas de trabalho/mês, distribuição de esforço entre etapas, entre outros), o CALICO calcula as equações ótimas para o projeto em questão.

O CALICO também deixa modificar quaisquer variáveis que definem um modelo de COCOMO, pode-se editar os multiplicadores de esforço, mudar as equações (conforme notado na figura 2), mudar as distribuições de esforço, entre outros.

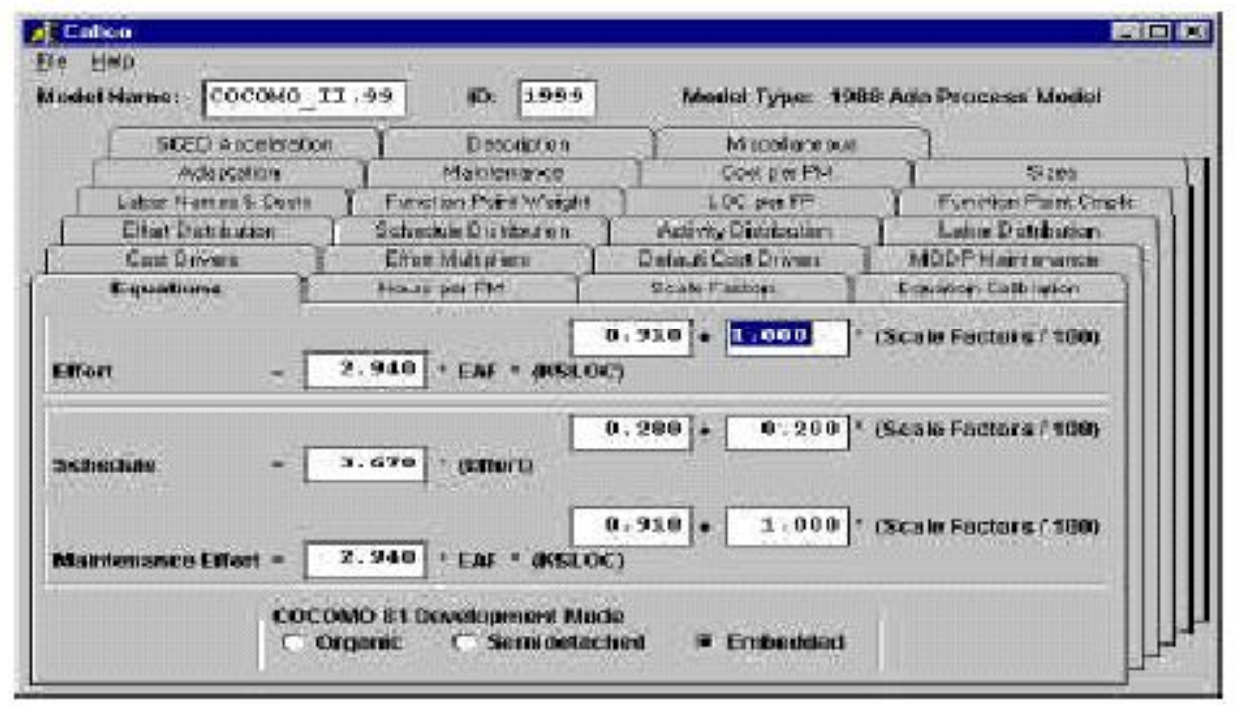

Figura 2 - Ferramenta Calico

Informações referentes a técnica de Análise de Ponto de Função são transformadas para linhas de código, conversão esta viabilizada através da informação da linguagem de programação utilizada.

O software não fornece recursos para visualização gráfica, e nem relatórios textuais, todas as informações advindas desta ferramenta são apenas visualizadas na tela.

\subsection{USC - COCOMO II}

O software USC-COCOMO II foi desenvolvido pela University of Southern Califórnia. A versão estudada teve sua última atualização recentemente no ano de 1999 . O USC COCOMO II, ilustrado na figura 3, é uma ferramenta CASE de análise que tem por fim gerar um resultado automatizado visando facilitar a administração de projetos de software. 


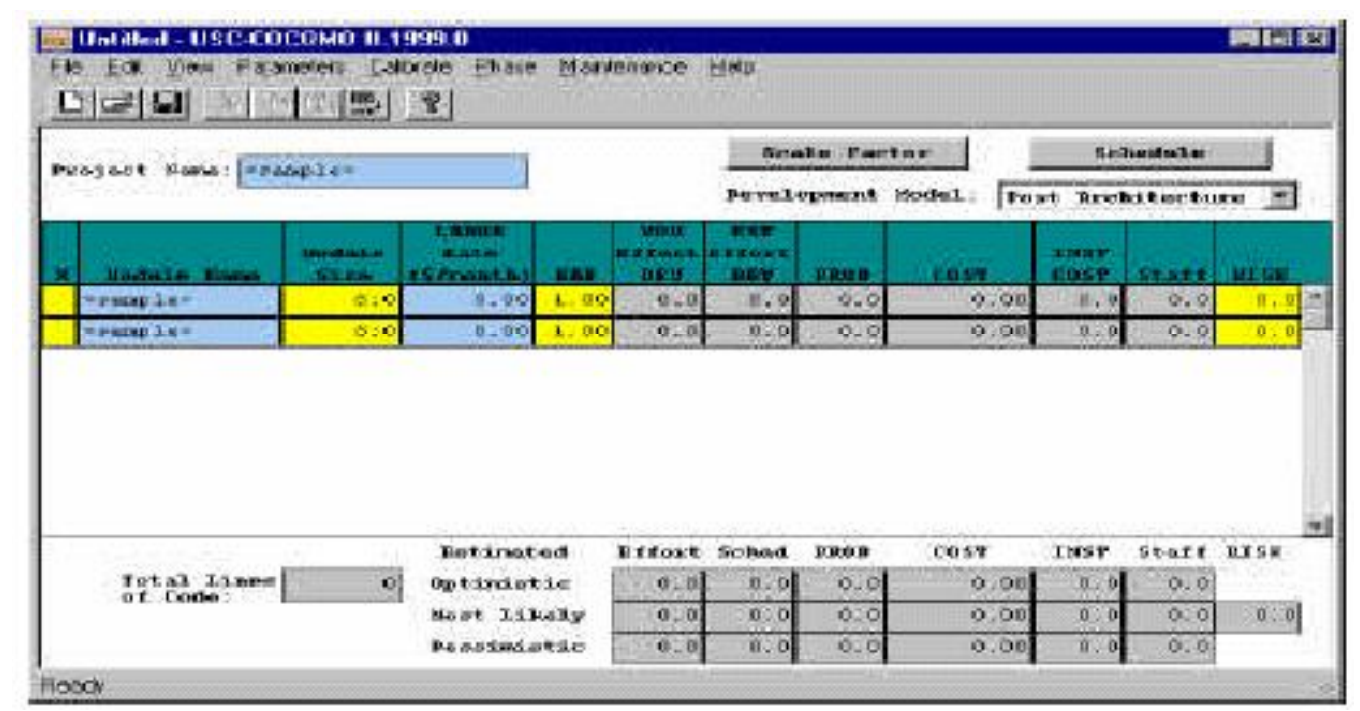

Figura 3 - Ferramenta USC-COCOMO II

USC-COCOMO estima o custo e tempo baseado em pessoa/mês e meses, respectivamente, para a determinação do baseline de exigências de um produto para a conclusão de uma atividade. O software prevê um adicional de $20 \%$ ao tempo computado, como margem de erro (análise de risco).

Para obter o custo por fase, dado que o COCOMO gera só o esforço/fase, é necessário gerar o custo médio por Pessoa-mês: calculado como custo e total para projeto inteiro (excluindo exigências) / Esforço Total em Pessoa-meses (excluindo exigências). A este cálculo é assumido um adicional de $7 \%$ do Esforço Total. O custo do projeto inteiro é a soma dos custos dos módulos individuais. Considerando que estas são só estimativas, são usadas tabelas para exibir o custo atual, gerando apenas uma estimativa Otimista e Pessimista para o custo do projeto inteiro. Para definir o tamanho do programa torna-se necessário que se caracterize que medida será adotada (linhas de código, pontos por função ou adaptação). A adaptação é a reformulação ou adaptação em um programa pré existente (as medidas por linhas de código e pontos por função foram abordadas na seção 3). O software implementa um método de calibração, aconselhado para menos de 8 projetos. Esta calibração realiza a análise dos dado que foram fornecidos ao programa e gera como resposta uma estimativa. Através desta ferramenta CASE pode-se também gerar pequenos relatórios textuais com estimativas vinculadas a cada fase do processo. As fases correspondem ao ciclo de vida clássico (modelo cascata) onde o esforço gasto em cada atividade é computado como uma porcentagem do esforço total consumido durante uma fase.

\subsection{Cost Xpert 2.1}

Cost Xpert 2.1. é um software desenvolvido para estimar tempo e custo relacionado com o desenvolvimento de projetos de software. O software pesquisado tem sua última versão lançada este ano pela empresa Marotz Inc., cujo site pode ser acessado através do endereço http://www.marotz.com, encontrando-se atualmente na versão 2.1. Para análise foi utilizada uma versão demo com licença de uso para 45 dias sem limitações uso. A interface do software pode ser visualizada na figura 4. 
O software mantêm informações gerais sobre o projeto e usuário responsável pelos dados informados. No que se refere a dados relativos ao projeto, é possível enquadrar o projeto em diferentes tipos e associa-lo a padrões e ciclos de vida, sendo que o software fornece abertura para se configurar os parâmetros pré-definidos.

Para estimar o tamanho do projeto pode-se utilizar sete métodos para cálculo: SLOC, Function Points, Feature Points, GUI Metrics, Object Métrics, Bottom Up e Top Down.

Cost Xpert 2.1 possui compatibilidade com o MS-project, pois permite criar Arquivos “.txt" e arquivo de dados de projeto que podem ser importados pelo Msproject e vice-versa.

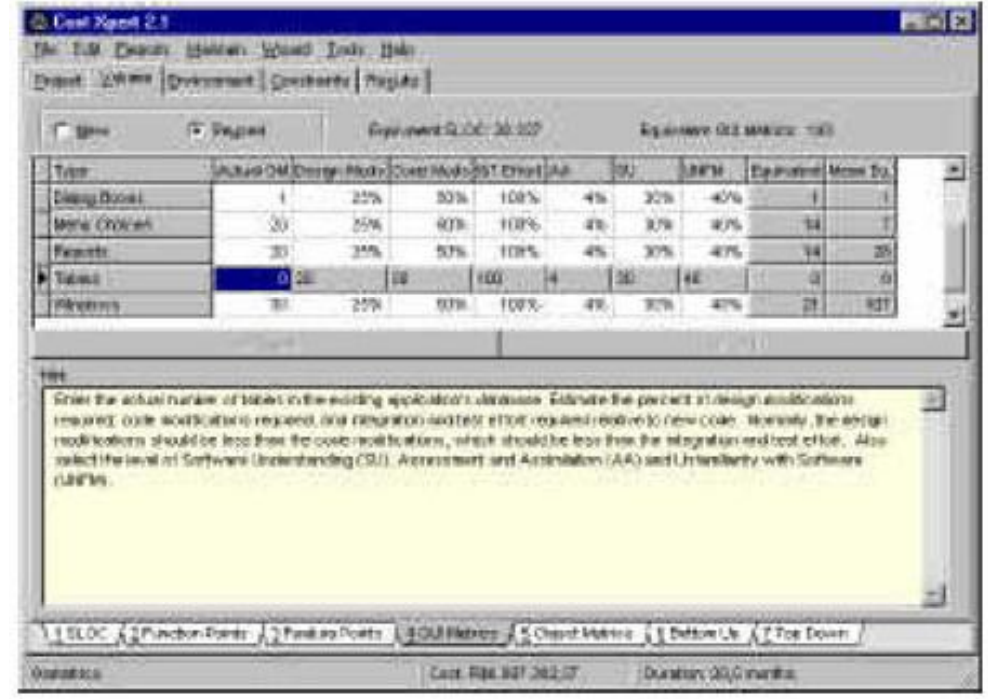

Figura 4 - Ferramenta Cost Xpert 2.1

O software realiza "análise de risco" calculando valores que afetam o esforço e também o tempo de entrega. Os fatores ambientais que podem afetar o custo global e duração de um projeto são divididos em categorias, tais como:

- Pessoal: considera fatores como experiência em análise, com o tipo de aplicação, linguagem e ferramenta, plataforma e programação.

- Plataforma: refere-se aos recursos para desenvolvimento.

- Projeto: considera ambiente e ferramentas disponíveis para desenvolvimento.

- Produto: refere-se a fatores relacionados com base de dados, documentação gerada durante o ciclo de vida, complexidade do produto, reusabilidade e tratamento a falhas.

-Fatores de escalonamento: considera fatores como riscos amenizados através do gerenciamento, flexibilidade dos requisitos, experiência de desenvolvimento do tipo de projeto, nível da empresa segundo CMM (Capability Maturity Model), interação da equipe (considerando equipe de desenvolvimento e também o stakeholder).

Cost Xpert 2.1 permite refinar a estimativa baseada em vários fatores. Entre os quais estão Tempo versus custo, esforço requerido para planejamento, esforço para testar e integrar o software (projeto), tempo para revisão por parte do cliente e contatos com o cliente para solucionar dúvidas e fatores relacionados com problemas eventuais.

Os resultados podem ser visualizados através de relatórios e também graficamente.

\section{Análise comparativa}




\begin{tabular}{|c|c|c|c|c|}
\hline & Calico & Costar & $\begin{array}{c}\text { USC- } \\
\text { COCOMO } \\
\text { II }\end{array}$ & Cost Xpert 2.1 \\
\hline \multirow[t]{2}{*}{ Linguagem por LOC } & $\begin{array}{c}\text { C, Fortran, } \\
\text { Cobol, Basic, } \\
\text { Pascal, Pl } / 1, \\
\text { Ada }\end{array}$ & $\begin{array}{c}\text { C, Fortran, } \\
\text { Cobol, Basic, } \\
\text { Pascal, Pl/1, Ada }\end{array}$ & $\begin{array}{l}\text { Vide lista } \\
\text { apresentada } \\
\text { abaixo } \\
\text { (linguagens } \\
\text { de } \\
\text { conversão } \\
\text { LOC }{ }^{*} \text { ) }\end{array}$ & $\begin{array}{l}\text { Inclui todas as } \\
\text { demais, } \\
\text { excluindo apenas } \\
\text { a linguagem } \\
\text { "Report } \\
\text { Generator" possui } \\
\text { mais } 500 \\
\text { incluindo } \\
\text { variação de } \\
\text { versão. }\end{array}$ \\
\hline & $\begin{array}{l}\text { Permite } \\
\text { inclusão de } \\
\text { nova } \\
\text { Linguagem }\end{array}$ & $\begin{array}{l}\text { Somente na } \\
\text { definição de um } \\
\text { novo modelo }\end{array}$ & $\begin{array}{c}\text { Não } \\
\text { permite } \\
\text { inclusão de } \\
\text { nova } \\
\text { linguagem }\end{array}$ & $\begin{array}{l}\text { Nào permite } \\
\text { inclusão de nova } \\
\text { linguagem }\end{array}$ \\
\hline Métricas/Indicadores & $\begin{array}{c}\mathrm{FP}, \\
\text { COCOMO I, } \\
\text { COCOMO } \\
\text { II }\end{array}$ & $\begin{array}{c}\text { FP, } \\
\text { COCOMO_II.97, } \\
\text { Early_Design, } \\
\text { COCOMO_85, } \\
\text { COCOMO_87, } \\
\text { Ada,87, Apm_88 }\end{array}$ & $\begin{array}{c}\text { FP, } \\
\text { Linhas de } \\
\text { código, } \\
\text { COCOMO } \\
\text { II }\end{array}$ & $\begin{array}{c}\text { FP, SLOC, } \\
\text { Feature Points, } \\
\text { GUI Metrics, } \\
\text { Object Metrics, } \\
\text { Bottom Up, Top } \\
\text { Down }\end{array}$ \\
\hline Processo & $\begin{array}{r}\text { Nắo } \\
\text { Contem }\end{array}$ & $\begin{array}{c}\text { Processo } \\
\text { iterativo (4 } \\
\text { variaçőes pré- } \\
\text { estabelecidas) }\end{array}$ & $\begin{array}{c}\text { Ciclo de } \\
\text { vida } \\
\text { Clássico } \\
\text { (Cascata) }\end{array}$ & Nào Contem \\
\hline Visualização Gráfica & $\begin{array}{c}\text { Năo } \\
\text { Contêm } \\
\text { Visualização } \\
\text { Gráfica }\end{array}$ & $\begin{array}{c}\text { Contêm } \\
\text { Visualização } \\
\text { Gráfica }\end{array}$ & $\begin{array}{c}\text { Nầo } \\
\text { Contêm } \\
\text { Visualização } \\
\text { Gráfica } \\
\end{array}$ & $\begin{array}{c}\text { Contêm } \\
\text { Visualização } \\
\text { Gráfica }\end{array}$ \\
\hline Relatórios & $\begin{array}{l}\text { Não emite } \\
\text { relatórios }\end{array}$ & $\begin{array}{c}\text { Emite } \\
\text { Relatórios com } \\
\text { gráficos }\end{array}$ & $\begin{array}{c}\text { Emite } \\
\text { relatórios } \\
\text { apenas } \\
\text { textuais }\end{array}$ & $\begin{array}{l}\text { Emite } \\
\text { Relatórios com } \\
\text { gráficos }\end{array}$ \\
\hline Recursos & $\begin{array}{c}\text { Esforço } \\
\text { (pessoa-Mês), } \\
\text { Duração } \\
\text { (pessoa-Mês) }\end{array}$ & $\begin{array}{c}\text { Esforço } \\
\text { (pessoa-Mês), } \\
\text { Custo, Duração } \\
\text { (Mês), }\end{array}$ & $\begin{array}{l}\text { Esforço } \\
\text { (pessoa- } \\
\text { Mês) } \\
\text { Custo, } \\
\text { Duração } \\
\text { (Mês), }\end{array}$ & $\begin{array}{c}\text { Pessoal, } \\
\text { Custos, Duração } \\
\text { (Meses) }\end{array}$ \\
\hline Help & Inexistente & $\begin{array}{l}\text { Documentação } \\
\text { On-Line (F1) }\end{array}$ & Manual & $\begin{array}{c}\text { Documentação } \\
\text { On-Line (F1), } \\
\text { sensivel ao } \\
\text { Contexto }\end{array}$ \\
\hline
\end{tabular}

Tabela 1 - Análise comparativa

Para a análise comparativa das ferramentas os critérios adotados foram:

- Linguagem por LOC: Quais as linguagens consideradas pela ferramenta para adaptação da quantidade de linhas de código, bem como se a ferramenta permite a inclusão de novas linguagens.

- Métricas/Indicadores: Quais as métricas ou indicadores implementados pela ferramenta.

- Processo: Processo de desenvolvimento adotado pela ferramenta (caso exista).

- Visualização Gráfica: Se a ferramenta gera algum gráfico comparativo para facilitar a análise dos resultados.

- Relatórios: Forma de apresentação dos relatórios (caso emita).

- Recursos: Quais os recursos cada ferramenta visa estimar?

- Help: Se a ferramenta apresenta alguma forma de documentação. 


\section{Considerações Finais}

Pode-se observar que as ferramentas CASE para métricas de software são poucas, e que possuem abrangência variável, ou seja, trabalham com conjunto de métricas diferentes, tendo-se como métricas amplamente aceitas os indicadores/métricas FPA, Linhas de código e algumas variações do COCOMO.

A finalidade das ferramentas também é diversa podendo tanto ser para geração de estimativas quanto para calibrar a equação utilizada pelo modelo COCOMO.

Pretende-se utilizar o resultado desta pesquisa para embaçar o projeto de pesquisa nas fases que se seguem, visando gerar uma ferramenta que se adeque as necessidades e anseios das empresas desenvolvedoras de software, e que, implementem técnicas amplamente aceitas.

\section{Referências}

BOMFIM, F. , AZEVEDO M. e HUDSON S. Métricas de Software On-Line: [ http://www.internext.com.br/mssa/ medidas.html]. ]. Disponível em 06 de abril de 2000.

BRAGA, A. Análise de Pontos de Função. Rio de Janeiro, Editora Infobook, 1996.

CANDÉAS, A., LOPES, C.. Estimativa: Uma ferramenta para agilizar o dimensionamento de projetos no SERPRO com base na metodologia de análise de pontos por função. XIII Simpósio Brasileiro de Engenharia de Software, Florianópolis, 1999.

International Function Point Users Group. On-Line: [http://www.ifpug.org/]. Disponível em 06 de abril de 2000.

PRESSMAN, R.. Engenharia de Software. São Paulo, Editora Makron Books, 1995.

Software Productivity Research. On-Line: [http://www.spr.com/]. Disponível em 05 de abril de 2000 .

\section{Informações bibliográficas:}

Conforme a NBR 6023:2002 da Associação Brasileira de Normas Técnicas (ABNT), este texto científico publicado em periódico eletrônico deve ser citado da seguinte forma:

FARIA JUNIOR, J. P. T. . Comparação entre Ferramentas CASE para gerenciamento de Projeto e Métricas de Software no Curso de Sistemas da Informação do UniFOA. Cadernos UniFOA, Volta Redonda, ano 1, n. 2, nov. 2006. Disponível em:

<http://www.unifoa.edu.br/pesquisa/caderno/edicao/02/11.pdf> 